\title{
ON BIFURCATION LOAD OF A THREE-MEMBER SLENDER SYSTEM WITH AN INTERNAL CRACK SUBJECTED TO EULER'S LOAD
}

\author{
Sebastian Uzny, Krzysztof Sokó£ \\ Częstochowa University of Technology, Institute of Mechanics and Machine Design Foundations, Częstochowa, Poland \\ e-mail:uzny@imipkm.pcz.pl; sokol@imipkm.pcz.pl
}

\begin{abstract}
The results of numerical simulations presented in this paper are concerned with instability of a three member slender system subjected to Euler load. The investigated column is built up as a flat frame composed of three rods. In the internal one, the defect is present in form of a crack. The boundary problem has been formulated on the basis of a static criterion of instability. The boundary conditions associated with different types of supports are obtained by proper selection of parameters of the generalized load. On the basis of these results, the magnitude of bifurcation load can be determined.
\end{abstract}

Keywords: column, divergence, conservative load, crack

\section{Introduction}

Numerical investigations on slender complex systems composed of beam elements with different rigidities have been presented in the papers (Godley and Chilver, 1970; Lueschen et al., 1996; Tomski and Uzny, 2008, Tomski et al.; 2014; Uzny, 2011a,b). In those papers, different types of conservative and non conservative loads have been considered. The most common type of a conservative load is the Euler load. The loading force has a constant line of action regardless of the deflection of the system. All known types of conservative loads can be expressed by means of the generalized load (Bochenek and Życzkowski, 2004; Gajewski and Życzkowski, 1970). It is induced by simultaneous actions of longitudinal and transversal forces and bending moments. The generalized load is a theoretical loading, because authors of the papers in which it is used do not present the design of loading heads. When the system is composed of elements with different bending and compression rigidities, the rectilinear and curvilinear forms of static equilibrium are present (Tomski and Uzny, 2008). The compressed system keeps the rectilinear form of static equilibrium up to the bifurcation load magnitude. An increase of the external load causes a change of the equilibrium form from the rectilinear into a curvilinear one. The maximum magnitude of the compressive force is called the critical one. If the constant total bending stiffness of the investigated system is used the change in relation of the bending stiffness between rods, it has no influence on critical load magnitude (Tomski et al., 2007). Only the change of bifurcation load can be observed because of asymmetry of the bending stiffness of the rods. The magnitude of the bifurcation load can be controlled by means of the following parameters: prestressing, additional elements responsible for transversal displacements, loading heads configuration - Tomski load (Tomski and Uzny, 2008; Uzny, 2011a). The change of the bifurcation load magnitude in relation to the rigidity of the system elements has influence on the occurrence of the local instability phenomenon (Tomski and Uzny, 2008; Uzny, 2011a,b). The rod with the lower rigidity is responsible for local instability (it deflects from the rectilinear form of static equilibrium and causes deflection of the whole system). In some cases, the rage of external load which corresponds to the curvilinear form of static equilibrium (from the bifurcation load 
magnitude up to the critical one) can be greater than the range of external load in the rectilinear form. The phenomenon of local instability of the discussed systems has a completely different nature than in thin walled structures.

In this paper, the instability of a complex system with consideration of the crack presence in the rod with the lowest rigidity is presented. Cracks in beam elements or columns have been investigated by many scientists (Anifantis and Dimarogonas, 1983; Chati et al., 1997; Kim and Kim, 2000; Krawczuk, 1992; Kukla, 2009; Masoud et al., 1999; Ostachowicz and Krawczuk, 1991; Sokól, 2014). In the literature, different methods of crack modeling can be found. The crack can be modeled as a rotational spring with properly chosen stiffness (Anifantis and Dimarogonas, 1983; Chati et al., 1997; Hjelmstad and Shin, 1996; Kim and Kim, 2000; Krawczuk, 1992; Masoud et al., 1999; Narkis, 1994; Ostachowicz and Krawczuk, 1991; Rizos et al., 1990; Shen and Taylor, 1991). In such a case, the system is composed of two beams or rods connected by a rotational spring in the point of crack location. The stiffness of the spring depends on relation of the crack depth to transverse dimensions and is being calculated with consideration of the fracture mechanics principles (Ostachowicz and Krawczuk, 199). Identification of characteristic parameters of cracks can be done on the basis of the vibration frequency, shape modes (Tomski et al., 2014) and the amplitude of vibration (Rizos et al., 1990).

A different method of crack modeling is presented in Chandros et al. (1998) according to which Crack Disturbance Functions are used. This method is more advanced because stress, strain and displacement are modified in the whole area of the damaged element (not only locally in the point of crack presence). The mentioned crack model is more accurate but at the same time more complex and time consuming. However, when the crack is being modelled by means of a rotational spring, good accuracy of the simulation and experimental results can be found (Chandros et al., 1998). On the basis of the analysis of the natural vibration frequency curves presented in (Chandros et al., 1998), it can be concluded that the difference between the two models (continuous cracked beam model and lumped crack flexibility model) is getting greater with an increase of the crack size. When small cracks are considered (crack smaller than half of the cross section), those differences are insignificant. Taking into account a comparison of experimental data with numerical simulations shown in (Chandros et al., 1998), there is a good foundation to use rotational springs in the modelling process of a small crack.

Zamorska et al. (2015) proposed analytical simulations done with the Green function of a cracked beam with a variable cross-sectional area and numerical results in CATIA software. Zhang et al. (2009) revealed a crack identification method by means of wavelet analysis with a transform matrix. While Ghadami et al. (2013) detected cracks with the use of natural frequencies.

The rotational spring has been used in (Sokół and Uzny, 2015) to simulate a crack in a multi-member slender system composed of three rods (symmetrically placed in relation to the axis of the system) in form of a flat frame. In the internal element, the crack was present. In that study, the investigations of the influence of crack location and size on the natural vibration frequency were done in systems with different boundary conditions subjected to the Euler load. The results presented in (Sokół and Uzny, 2015) can be used in a diagnostic process of supporting systems.

In this paper, a continuation of studies started in (Sokół and Uzny, 2015) is presented. On the basis of those results, it has been stated that detailed studies on the bifurcation load should be done because of presence of discontinuity of curves that describe the change of static equilibrium form. The results shown here are focused on the bifurcation load magnitude at which the change of form of static equilibrium from a rectilinear into curvilinear one takes place. The influence of the crack size and relation of elasticity moduli is also taken into account. 


\section{Boundary problem formulation}

The considered slender system (Fig. 1a) is subjected to a compressive external load with a constant line of action. The column is composed of elements (rods) with different rigidities which are symmetrically placed relative to the axis of the system.

Two external rods have equal rigidities while the third one is characterized by a smaller rigidity relative to the others. Additionally, in the internal element, a defect in form of a crack (slit) is investigated. In this paper, results of numerical simulations for different boundary conditions are presented (Fig. $1 \mathrm{~b}-E U i$ where $i$ stands for a different type of support 1-5).
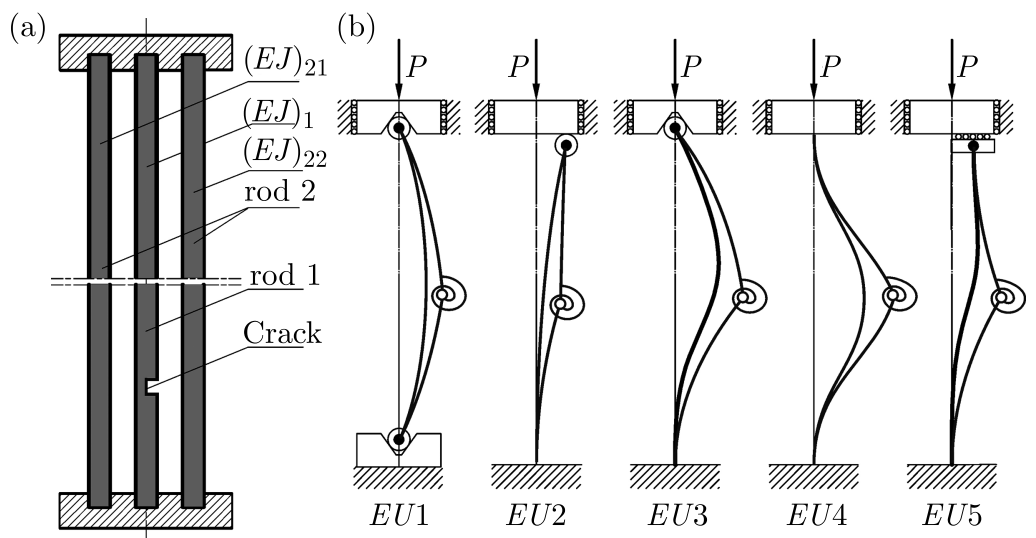

Fig. 1. Investigated multi-member system: (a) physical model of a flat frame with a marked defect crack, (b) schematic diagrams of the system with different boundary conditions and the installed rotational spring in the point of crack presence

In the problem formulation, a generalized load (see Bochenek and Życzkowski, 2004; Gajewski and Życzkowski, 1970) on both ends of the column is used. The advantage of the theoretical generalized load is that it can be implemented in order to show all known types of conservative loads (therein the Euler load in the system with different boundary conditions). The applied force is a longitudinal external force $P$. The $H_{k}$ and $M_{k}$ components are generalized forces which arise due to the action of $P$. The index $k$ is introduced and it can take values: 0 for $x_{21}=0$ and 1 for $x_{21}=l_{21}$.

In the boundary problem formulation process, the four rods are taken into account (Fig. 2) with rigidities $E_{11}, E_{12}, E_{21}, E_{22}$. Elements 21 and 22 have equal rigidities, while 11 and 22 stand for the cracked element. The crack is simulated by means of a rotational spring of stiffness $C$ and linear characteristics in the point of connection of rods 11 and 12 . The function $W_{i j}\left(x_{i j}\right) i, j=1,2$ describes transversal displacements of the rods at a given coordinate $x_{i j}$. The components $H_{k}$ and $M_{k}$ of the generalized load depend on the compressive force $P$ and can be expressed in form (see Gajewski and Życzkowski, 1970)

$$
\begin{aligned}
& H_{k}=P\left[-\left.(-1)^{k}\left(1-\varphi_{k}\right) \frac{d W_{21}\left(x_{21}\right)}{d x_{21}}\right|^{x_{21}=k l_{21}}-\gamma_{k} W_{21}\left(k l_{21}\right)\right] \\
& M_{k}=P\left[-\left.(-1)^{k} \rho_{k} \frac{d W_{21}\left(x_{21}\right)}{d x_{21}}\right|^{x_{21}=k l_{21}}+\nu_{k} W_{21}\left(k l_{21}\right)\right]
\end{aligned}
$$

where $\phi_{k}, \gamma_{k}, \rho_{k}, \nu_{k}$ are the coefficients which depend on the type of support or loading head. The magnitudes of $\phi_{k}, \gamma_{k}, \rho_{k}, \nu_{k}$ for different types of support with consideration of Euler load are presented in Table 1.

The boundary problem formulation is done by means of the static criterion of instability

$$
\delta V=0
$$




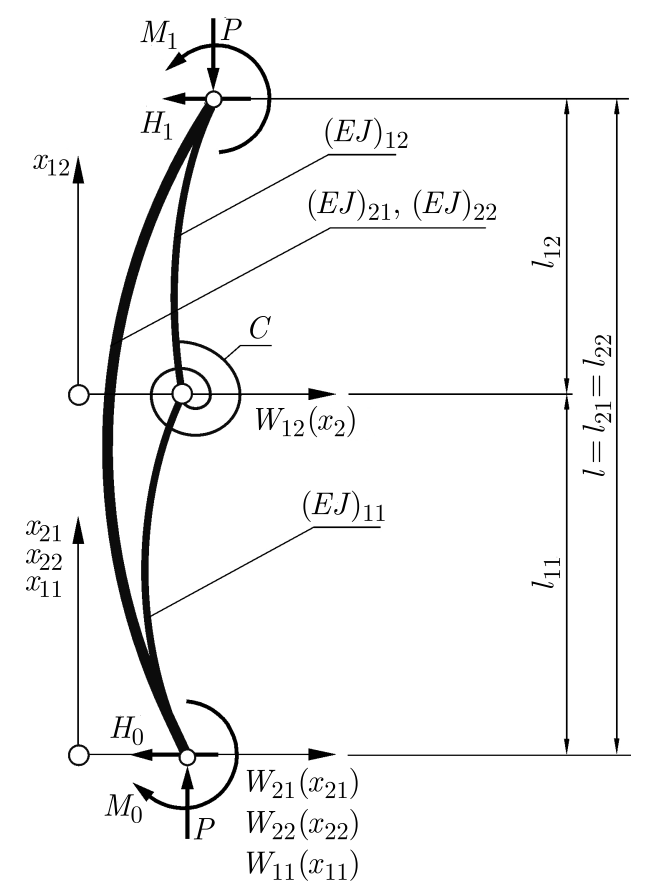

Fig. 2. Calculation model of the considered system subjected to the generalized load on both ends

Table 1. Magnitudes of generalized load parameters

\begin{tabular}{|c|c|c|c|c|c|}
\cline { 2 - 6 } \multicolumn{1}{c|}{} & \multicolumn{5}{c|}{ Type of suport } \\
\cline { 2 - 6 } \multicolumn{1}{c|}{} & $E U 1$ & $E U 2$ & $E U 3$ & $E U 4$ & $E U 5$ \\
\hline \hline$\phi_{0}$ & 1 & 1 & 1 & 1 & 1 \\
\hline$\gamma_{0}$ & $1 / \gamma_{0}=0$ & $1 / \gamma_{0}=0$ & $1 / \gamma_{0}=0$ & $1 / \gamma_{0}=0$ & $1 / \gamma_{0}=0$ \\
\hline$\rho_{0}$ & 0 & $1 / \rho_{0}=0$ & $1 / \rho_{0}=0$ & $1 / \rho_{0}=0$ & $1 / \rho_{0}=0$ \\
\hline$\nu_{0}$ & 0 & 1 & 1 & 1 & 1 \\
\hline$\phi_{1}$ & 1 & 1 & 1 & 1 & 0 \\
\hline$\gamma_{1}$ & $1 / \gamma_{1}=0$ & 0 & $1 / \gamma_{1}=0$ & $1 / \gamma_{1}=0$ & 0 \\
\hline$\rho_{1}$ & 0 & 0 & 0 & $1 / \rho_{1}=0$ & $1 / \rho_{1}=0$ \\
\hline$\nu_{1}$ & 0 & 0 & 0 & 1 & 1 \\
\hline
\end{tabular}

on the basis of which the estimation of the magnitude of the bifurcation load can be done. The potential energy of the system shown in Fig. 2 is as follows

$$
\begin{aligned}
V= & \frac{1}{2} C\left[\left.\frac{d W_{11}\left(x_{11}\right)}{d x_{11}}\right|^{x_{11}=l_{11}}-\left.\frac{d W_{12}\left(x_{12}\right)}{d x_{12}}\right|_{x_{12}=0}\right]^{2}+\frac{1}{2} \sum_{i=1}^{2} \sum_{j=1}^{2}(E J)_{i j} \int_{0}^{l_{i j}}\left[\frac{d^{2} W_{i j}\left(x_{i j}\right)}{d x_{i j}^{2}}\right]^{2} d x_{i j} \\
& +\frac{1}{2} \sum_{i=1}^{2} \sum_{j=1}^{2}(E A)_{i j} \int_{0}^{l_{i j}}\left[\frac{d U_{i j}\left(x_{i j}\right)}{d x_{i j}}+\frac{1}{2}\left(\frac{d W_{i j}\left(x_{i j}\right)}{d x_{i j}}\right)^{2}\right]^{2} d x_{i j}+P U_{21}\left(l_{21}\right) \\
& +\sum_{i=0}^{1} \frac{1}{2}\left\{-\left.(-1)^{i} P\left[-\left.(-1)^{i} \rho_{i} \frac{d W_{21}\left(x_{21}\right)}{d x_{21}}\right|^{x_{21}=i l}+\nu_{i} W_{21}(i l)\right] \frac{d W_{21}\left(x_{21}\right)}{d x_{21}}\right|_{x_{21}=i l_{21}}\right. \\
& \left.+P\left[-\left.(-1)^{i}\left(1-\varphi_{i}\right) \frac{d W_{21}\left(x_{21}\right)}{d x_{21}}\right|^{x_{21}=i l}-\gamma_{i} W_{21}(i l)\right] W_{21}(i l)\right\}
\end{aligned}
$$


Geometrical boundary conditions can be written in form

$$
\begin{aligned}
& U_{11}(0)=U_{21}(0)=U_{22}(0)=0 \quad U_{12}\left(l_{12}\right)=U_{21}\left(l_{21}\right)=U_{22}\left(l_{22}\right) \\
& U_{11}\left(l_{11}\right)=U_{12}(0) \quad W_{11}(0)=W_{21}(0)=W_{22}(0) \\
& W_{12}\left(l_{12}\right)=W_{21}\left(l_{21}\right)=W_{22}\left(l_{22}\right) \quad W_{11}\left(l_{11}\right)=W_{12}(0) \\
& \left.\frac{d W_{11}\left(x_{11}\right)}{d x_{11}}\right|_{x_{11}=0}=\left.\frac{d W_{21}\left(x_{21}\right)}{d x_{21}}\right|_{x_{21}=0}=\left.\frac{d W_{22}\left(x_{22}\right)}{d x_{22}}\right|_{x_{22}=0} \\
& \left.\frac{d W_{12}\left(x_{12}\right)}{d x_{12}}\right|^{x_{11}=l_{12}}=\left.\frac{d W_{21}\left(x_{21}\right)}{d x_{21}}\right|^{x_{21}=l_{21}}=\left.\frac{d W_{22}\left(x_{22}\right)}{d x_{22}}\right|^{x_{22}=l_{22}}
\end{aligned}
$$

Introduction of potential energy (2.3) into (2.2) leads to equations of transversal and longitudinal displacements and natural boundary conditions on each end of the system at $x_{11}=x_{21}=x_{22}=$ $0 ; x_{12}=l_{12}, x_{21}=l_{21}, x_{22}=l_{22}$ and crack location $x_{11}=l_{11}, x_{12}=0$.

The differential equation of transversal displacements is as follows

$$
(E J)_{i j} \frac{d^{4} W_{i j}\left(x_{i j}\right)}{d x_{i j}^{4}}+S_{i j} \frac{d^{2} W_{i j}\left(x_{i j}\right)}{d x_{i j}^{2}}=0
$$

While the longitudinal displacements can be presented in form

$$
U_{i j}\left(x_{i j}\right)-U_{i j}(0)=-\frac{S_{i j}}{(E A)_{i j}} x_{i j}-\int_{0}^{x_{i j}}\left(\frac{d W_{i j}\left(x_{i j}\right)}{d x_{i j}}\right)^{2} d x_{i j} \quad \begin{aligned}
& i=1,2 \\
& j=1,2
\end{aligned}
$$

Natural boundary conditions are shown below

$$
\begin{aligned}
& \left.(E J)_{11} \frac{d^{3} W_{11}\left(x_{11}\right)}{d x_{11}^{3}}\right|_{x_{11}=0}+\left.\sum_{i=1}^{2}(E J)_{2 i} \frac{d^{3} W_{2 i}\left(x_{2 i}\right)}{d x_{2 i}^{3}}\right|_{x_{2 i}=0}+\left.S_{11} \frac{d W_{11}\left(x_{11}\right)}{d x_{11}}\right|_{x_{11}=0} \\
& +\left.\sum_{i=1}^{2} S_{2 i} \frac{d W_{2 i}\left(x_{2 i}\right)}{d x_{2 i}}\right|_{x_{2 i}=0}-P\left[\gamma_{0} W_{21}(0)+\left.\left(1-\varphi_{0}\right) \frac{d W_{21}\left(x_{21}\right)}{d x_{21}}\right|_{x_{21}=0}\right]=0 \\
& \left.(E J)_{11} \frac{d^{2} W_{11}\left(x_{11}\right)}{d x_{11}^{2}}\right|_{x_{11}=0}+\left.\sum_{i=1}^{2}(E J)_{2 i} \frac{d^{2} W_{2 i}\left(x_{2 i}\right)}{d x_{2 i}^{2}}\right|_{x_{2 i}=0} \\
& +P\left[\nu_{0} W_{21}(0)-\left.\rho_{0} \frac{d W_{21}\left(x_{21}\right)}{d x_{21}}\right|_{x_{21}=0}\right]=0 \\
& \left.(E J)_{12} \frac{d^{3} W_{12}\left(x_{12}\right)}{d x_{12}^{3}}\right|^{x_{12}=l_{12}}+\left.\sum_{i=1}^{2}(E J)_{2 i} \frac{d^{3} W_{2 i}\left(x_{2 i}\right)}{d x_{2 i}^{3}}\right|^{x_{2 i}=l_{2 i}}+\left.S_{12} \frac{d W_{12}\left(x_{12}\right)}{d x_{12}}\right|^{x_{12}=l_{12}} \\
& +\left.\sum_{i=1}^{2} S_{2 i} \frac{d W_{2 i}\left(x_{2 i}\right)}{d x_{2 i}^{3}}\right|^{x_{2 i}=l_{2 i}}+P\left[\gamma_{1} W_{21}\left(l_{21}\right)-\left.\left(1-\varphi_{1}\right) \frac{d W_{21}\left(x_{21}\right)}{d x_{21}}\right|^{x_{21}=l_{21}}\right]=0 \\
& \left.\left.(E J)_{11} \frac{d^{2} W_{11}\left(x_{11}\right)}{d x_{11}^{2}}\right|^{x_{12}=l_{12}}+\left.\sum_{i=1}^{2}(E J)_{2 i} \frac{d^{2} W_{2 i}\left(x_{2 i}\right)}{d x_{2 i}^{2}}\right|^{x_{2 i}=l_{2 i}}\right]=0 \\
& +P\left[\nu_{1} W_{21}\left(l_{21}\right)+\left.\rho_{1} \frac{d W_{21}\left(x_{21}\right)}{d x_{21}}\right|^{x_{21}=l_{21}}\right]=0 \\
& \left.(E J)_{11} \frac{d^{2} W_{11}\left(x_{11}\right)}{d x_{11}^{2}}\right|^{x_{11}=l_{11}}+C_{R}\left[\left.\frac{d W_{11}\left(x_{11}\right)}{d x_{11}}\right|^{x_{11}=l_{11}}-\left.\frac{d W_{12}\left(x_{12}\right)}{d x_{12}}\right|_{x_{12}=0}\right]=0
\end{aligned}
$$




$$
\begin{aligned}
& \left.(E J)_{12} \frac{d^{2} W_{12}\left(x_{12}\right)}{d x_{12}^{2}}\right|_{x_{12}=0}+C_{R}\left[\left.\frac{d W_{11}\left(x_{11}\right)}{d x_{11}}\right|^{x_{11}=l_{11}}-\left.\frac{d W_{12}\left(x_{12}\right)}{d x_{12}}\right|_{x_{12}=0}\right]=0 \\
& \left.\sum_{i=1}^{2}(E J)_{1 i}(-1)^{i+1} \frac{d^{3} W_{1 i}\left(x_{1 i}\right)}{d x_{1 i}^{3}}\right|^{x_{1 i}=\left(l_{1 i}\right)(2-i)}+\left.S_{11} \frac{d W_{11}\left(x_{11}\right)}{d x_{11}}\right|^{x_{11}=l_{11}} \\
& \quad-\left.S_{12} \frac{d W_{12}\left(x_{12}\right)}{d x_{12}}\right|_{x_{12}=0}=0
\end{aligned}
$$

and

$$
S_{12}+S_{21}+S_{22}-P=0
$$

Complex slender systems are characterized by the presence of rectilinear and curvilinear form of static equilibrium. In this paper, the rectilinear form is only considered. In this case, the internal force $S_{i j}$ in the element is obtained on the basis of the following relations

$$
S_{11}=S_{12} \quad S_{21}=S_{22} \quad S_{12}=P \frac{(E A)_{12}}{(E A)_{12}}+2(E A)_{21} \quad S_{22}=\frac{P-S_{12}}{2}
$$

The relations between internal forces have been calculated with consideration of boundary conditions (2.4) and (2.8). The presented method of formulation of the boundary problem in which the theoretical generalized load is used shows that numerical simulations can be performed for different types of supports and loads (including Tomski load (see Tomski and Uzny, 2008; Tomski et al., 2007, 2014)). In the boundary conditions, only the parameters $\rho_{k}, \nu_{k}, \phi_{k}, \gamma_{k}$, where $k=0,1$, must be introduced.

The solution of the differential equations of transversal displacements has been performed on the basis of the following expression, $i=1,2$ and $j=1,2$

$$
W_{i j}\left(x_{i j}\right)=A_{i j} \exp \left(\mathrm{i} \sqrt{P} x_{i j}\right)+B_{i j} \exp \left(-\mathrm{i} \sqrt{P} x_{i j}\right)+C_{i j} x_{i j}+D_{i j}
$$

After introduction of (2.10) into the boundary conditions, one obtains a system of equations for which the matrix determinant equated to zero creates a transcendental equation used for the estimation of the bifurcation load.

\section{Results of numerical simulations}

The results of numerical simulations performed on the basis of the proposed mathematical model have been presented in the plane bifurcation load - flexural rigidity asymmetry factor $\mu_{a}$. The coefficient $\mu_{a}$ is defined as a relation of the flexural rigidity of the cracked rod to the sum of rigidities of the external rods

$$
\mu_{a}=\frac{(E J)_{11}}{(E J)_{21}+(E J)_{22}}
$$

The magnitude of bifurcation load is presented in the non-dimensional form

$$
\lambda_{b}=\frac{P l_{21}^{2}}{(E J)_{11}}+\sum_{i=1}^{2}(E J)_{2 i}
$$

In simulations, a constant total flexural rigidity of the system is used

$$
(E J)_{11}+(E J)_{21}+(E J)_{22}=E J
$$


Numerical simulations have been performed for three magnitudes of the parameter $\zeta_{B}$ $\left(\zeta_{B}=0.1,0.5,1.0\right)$. The $\zeta_{B}$ shows the relation between the Young modulus of the cracked rod to the uncracked one

$$
\zeta_{B}=\frac{E_{11}}{E_{21}}
$$

The non-dimensional spring stiffness parameter $c$ which reflects the crack size and the location $\zeta_{A}$ are as follows

$$
c=\frac{C l}{E J} \quad \zeta_{A}=\frac{l_{11}}{l}
$$

The results presented in this paper are done only at $\zeta_{A}=0.5$ (central location).

(a)

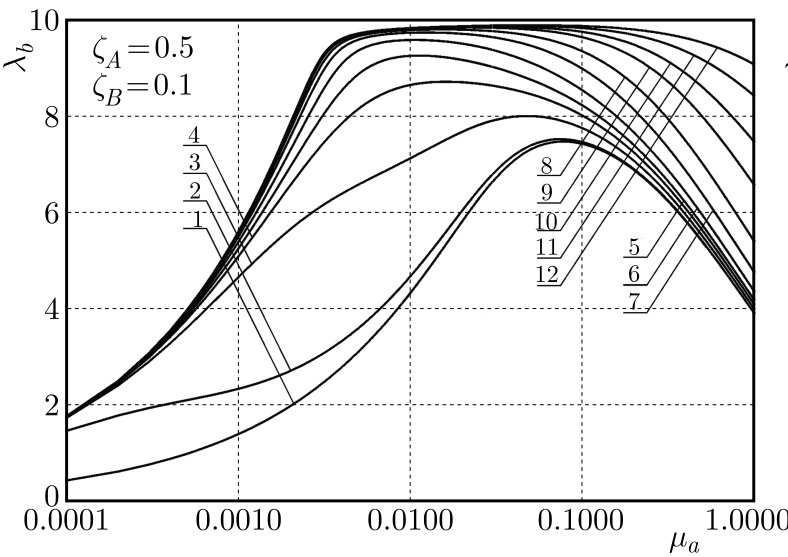

(b)

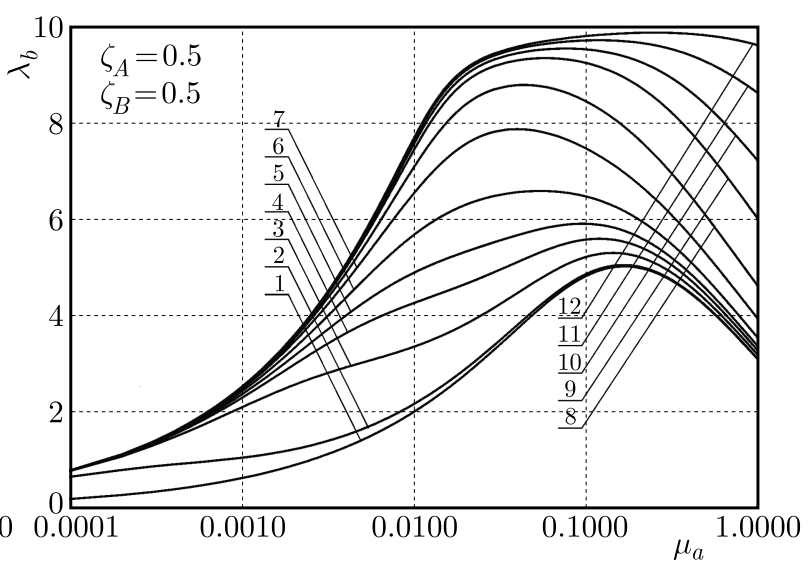

(c)
1. $c=0$
2. $c=0.001$
3. $c=0.010$
4. $c=0.020$
5. $c=0.030$
6. $c=0.050$
7. $c=0.100$
8. $c=0.200$
9. $c=0.500$
10. $c=1.000$
11. $c=3.000$
12. $1 / c=0$

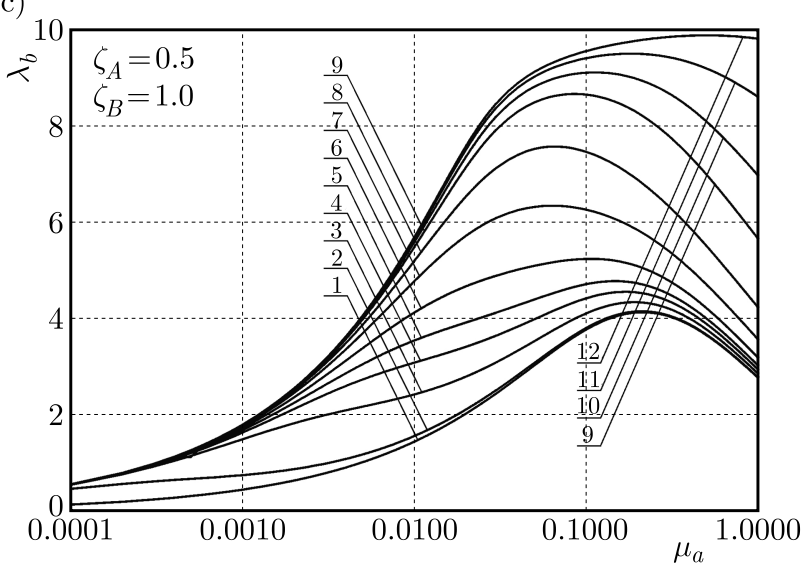

Fig. 3. A change of the bifurcation load parameter $\lambda_{b}$ of $E U 1$ column in relation to the flexural rigidity asymmetry factor $\mu_{a}$ at $\zeta_{A}=0.5$ and different crack sizes: (a) $\zeta_{B}=0.1$, (b) $\zeta_{B}=0.5$, (c) $\zeta_{B}=1$

On the basis of the results of numerical simulations presented in Figs. 3-7 it have been shown that the crack size has a small influence on the bifurcation load magnitude at a lower level of the bending rigidity asymmetry factor. This influence depends on the boundary conditions and Young modulus of the materials used in the supporting structure. When the materials are comparable (which corresponds to $\zeta_{B} \approx 1$ ) the differences in bifurcation loads are the smallest at low $\mu_{a}$. An increase in the bending rigidity asymmetry factor $\mu_{a}$ causes an increase in bifurcation load to the maximum level above which a further increase of $\mu_{a}$ results in reduction of the loading 
(a)

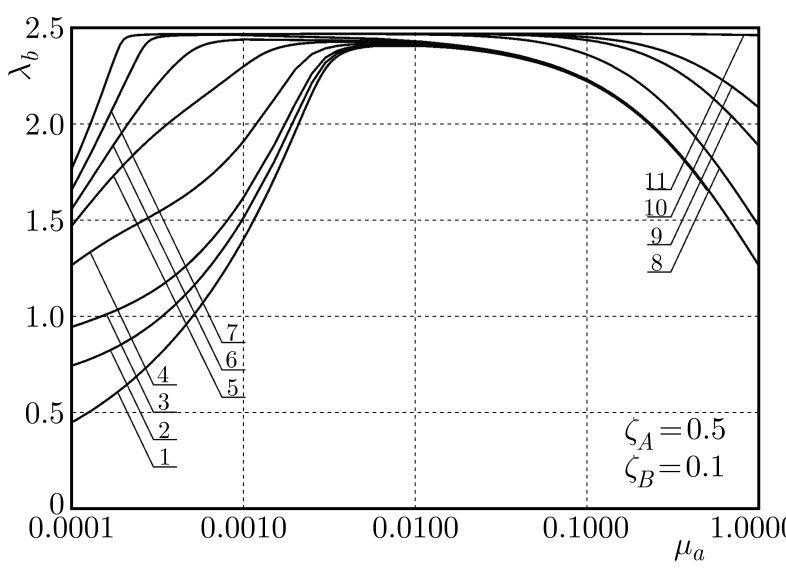

1. $c=0$

2. $c=0.0001$

3. $c=0.0002$

4. $c=0.0005$

5. $c=0.0010$

6. $c=0.0015$

7. $c=0.0030$

8. $c=0.1000$

9. $c=0.5000$

10. $c=1.000$

11. $1 / c=0$ (b)

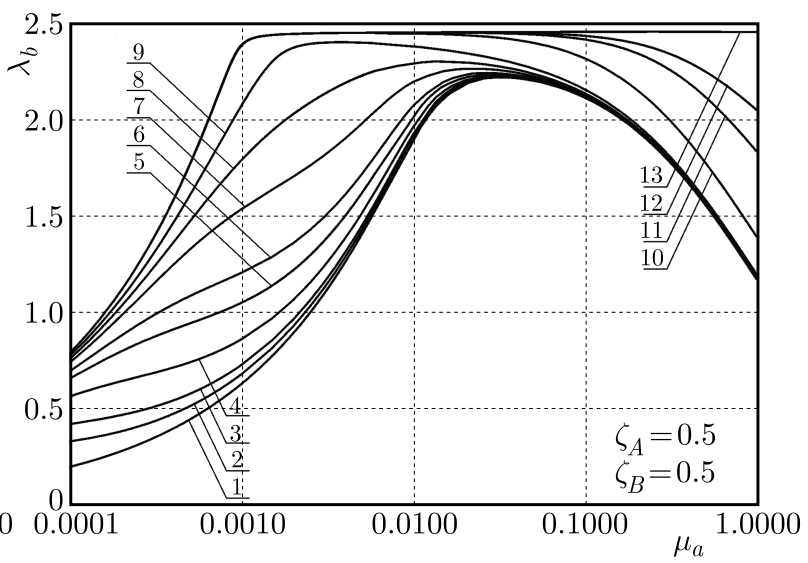

(c)
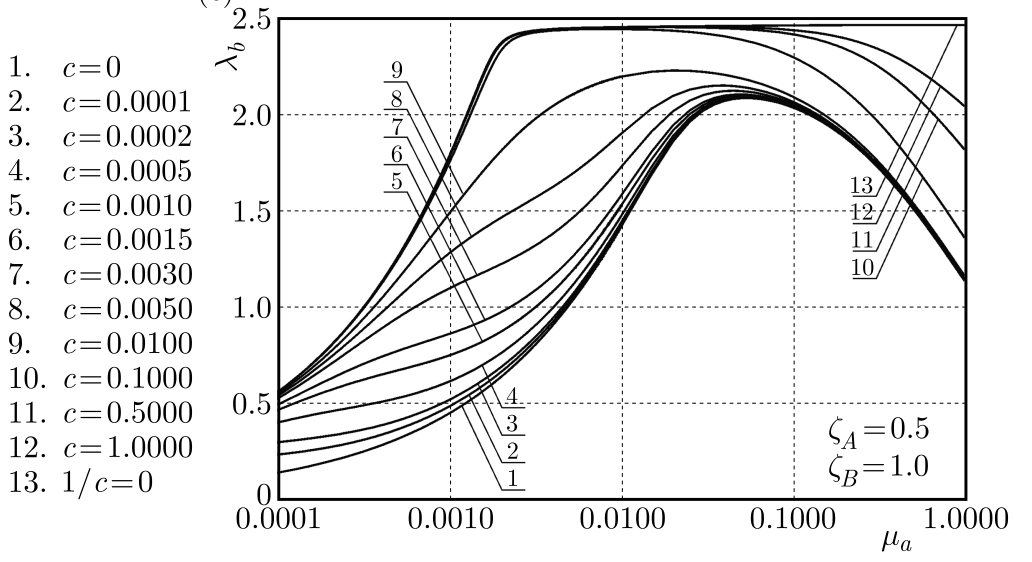

Fig. 4. A change of the bifurcation load parameter $\lambda_{b}$ of $E U 2$ column in relation to the flexural rigidity asymmetry factor $\mu_{a}$ at $\zeta_{A}=0.5$ and different crack sizes: (a) $\zeta_{B}=0.1$, (b) $\zeta_{B}=0.5$, (c) $\zeta_{B}=1$

capacity. In EU4 and EU5 configurations at higher $\mu_{a}$, the bifurcation load is constant and independent of the crack size. Furthermore, in EU5 the area of independency of the bifurcation load to $\mu_{a}$ can be found (the smaller crack, the greater the independency area). On the basis of the numerical simulations, it can be concluded that columns EU4 and EU5 at higher $\mu_{a}$ are insensitive to the crack size, which appears in the central element (the one with lower bending rigidity). In all investigated cases such ranges of the crack size can be estimated at which a small change of the bifurcation load regardless of $\mu_{a}$ can be found.

Additionally, it has been shown that in configurations EU4 and EU5, the discontinuity of the curves can be observed in the plane loading parameter - flexural rigidity asymmetry factor $\lambda_{b}\left(\mu_{a}\right)$. In columns $E U 4$ and $E U 5$, at a sufficiently high flexural rigidity asymmetry factor, a change in buckling mode shapes takes place. The magnitude of this factor at which the change can be observed highly depends on the crack size (rotational spring stiffness $c$ ). The smaller crack, the higher $\mu_{a}$ is needed to obtain a change in the buckling mode shape. The buckling mode shapes of the considered systems are presented in Fig. 8. A high difference in the parameter $\mu_{a}$ has been chosen in order to achieve the best presentation of the change of buckling shapes, especially for EU4 and EU5.

In configurations $E U 1-E U 3$, an increase of $\mu_{a}$ reveals the presence of the crack. That is why in those three cases an observation of buckling shape modes can easily lead to determination of failure of the structure at high $\mu_{a}$ because the function used to describe the transversal displacements of the cracked element is not a smooth one. The buckling shape modes of EU4 and EU5 systems at a lower magnitude of the $\mu_{a}$ coefficient are characterized by no transversal 
(a)

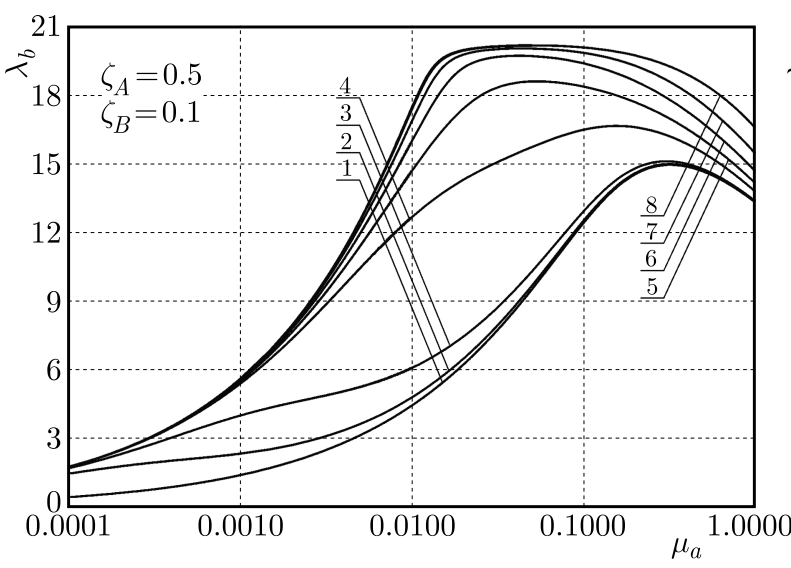

(b)

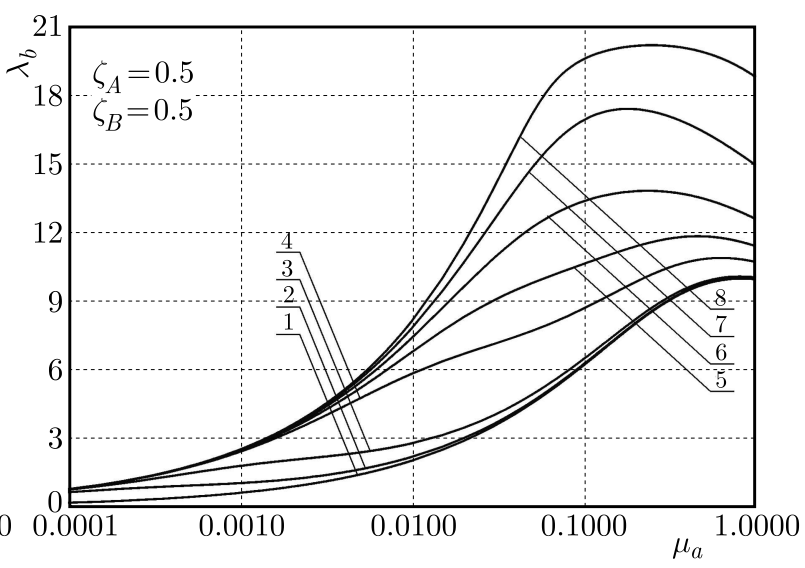

(c)

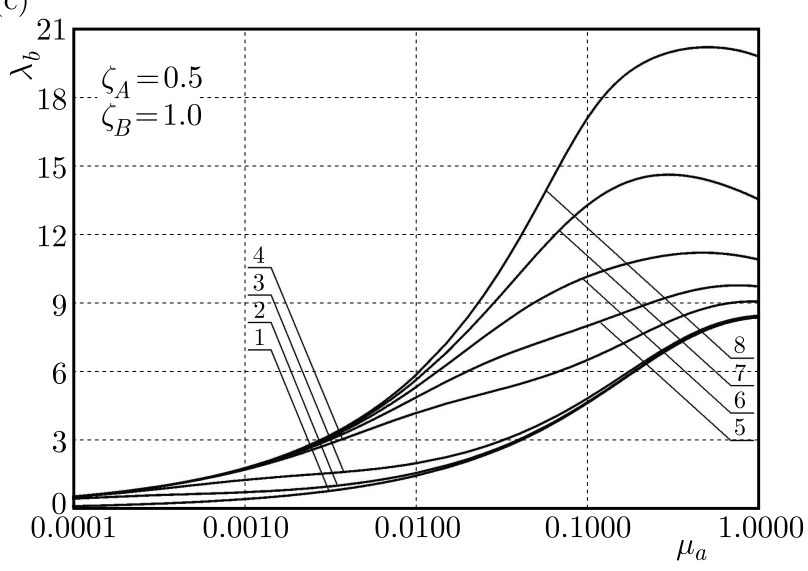

Fig. 5. A change of the bifurcation load parameter $\lambda_{b}$ of $E U 3$ column in relation to the flexural rigidity asymmetry factor $\mu_{a}$ at $\zeta_{A}=0.5$ and different crack sizes: (a) $\zeta_{B}=0.1$, (b) $\zeta_{B}=0.5$, (c) $\zeta_{B}=1$

displacement of the uncracked rods (see Fig. 8 - shapes at $\mu_{a}=0.01-E U 4$ and $\mu_{a}=0.001$ - EU5 configurations). At $\mu_{a}=0.9$, the buckling shape modes of EU4 and EU5 are changing regarding to lower $\mu_{a}$. The change is related to independency of the bifurcation load of the considered systems from the crack size at higher $\mu_{a}$ magnitudes. Additionally, when columns EU4 and EU5 are taken into account, the buckling shape modes are described by a smooth function. In EU4 and EU5 configurations, the crack presence is hard to identify on the basis of analysis of buckling shape modes. That is why, the further investigations on the natural vibration frequency must be done (relations: external load-vibration frequency and amplitude-vibration frequency). The solution presented in this paper allows one to choose proper physical and geometrical parameters and reveals the area of drop in the loading capacity in the case of crack presence.

\section{Conclusions}

In this paper, a slender system with a crack subjected to compressive external load with a constant line of action is considered. The effect of size of the crack, which is present in the internal element, on the bifurcation load magnitude is investigated. Numerical simulations of the bifurcation load have been done at different magnitudes of parameters such as: rotational spring stiffness (size of the crack), flexural rigidity asymmetry factor and longitudinal elasticity modulus. The numerical calculations are also concerned with different types of supports (five configurations have been chosen for presentation). 
(a)

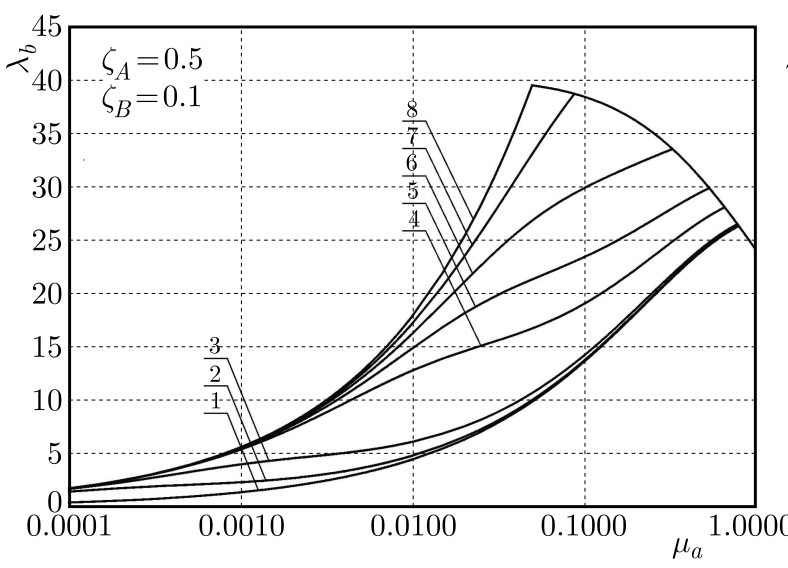

(b)

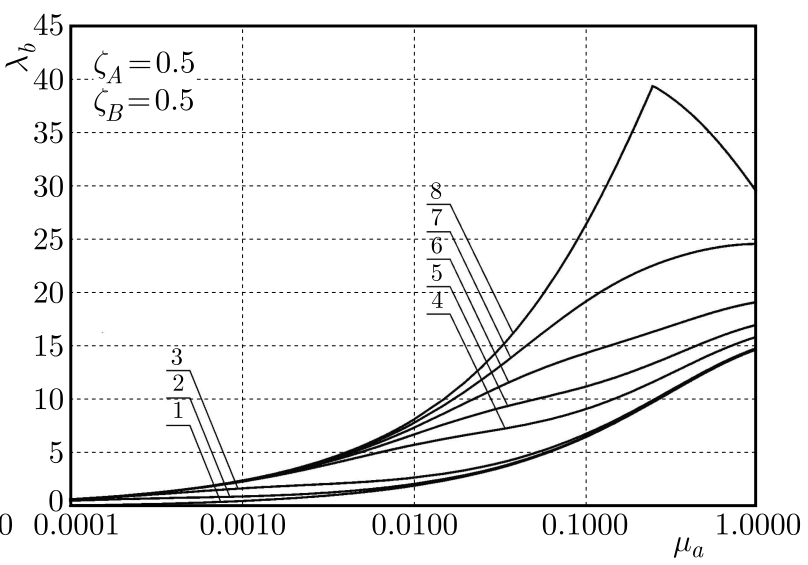

(c)

$$
\begin{array}{ll}
\text { 1. } & c=0 \\
\text { 2. } & c=0.001 \\
\text { 3. } & c=0.005 \\
\text { 4. } & c=0.050 \\
\text { 5. } & c=0.100 \\
\text { 6. } & c=0.200 \\
\text { 7. } & c=0.500 \\
\text { 8. } & 1 / c=0
\end{array}
$$

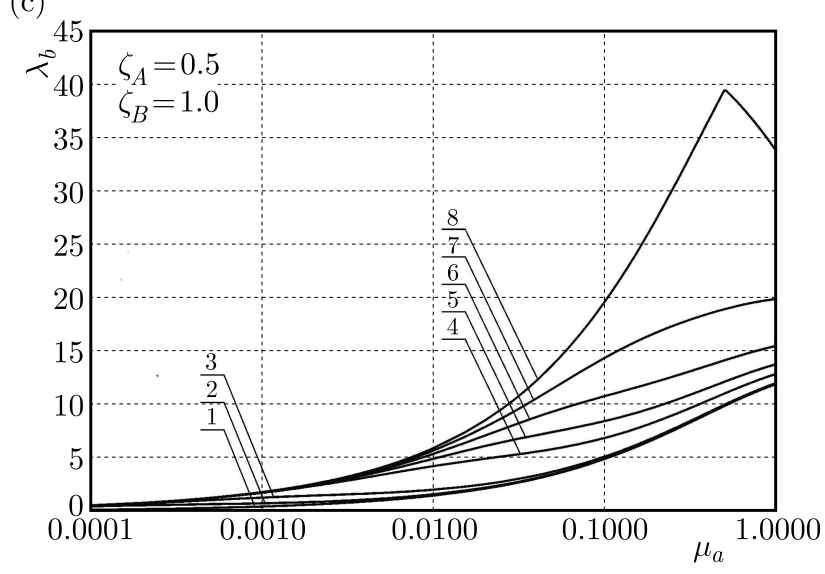

Fig. 6. A change of the bifurcation load parameter $\lambda_{b}$ of $E U 4$ column in relation to the flexural rigidity asymmetry factor $\mu_{a}$ at $\zeta_{A}=0.5$ and different crack sizes: (a) $\zeta_{B}=0.1$, (b) $\zeta_{B}=0.5$, (c) $\zeta_{B}=1$

On the basis of the results of simulations, the following conclusions can be drawn:

- influence of the crack size on the bifurcation load magnitude highly depends on a combination of the supporting elements (boundary conditions),

- in the systems with the zero deflection angle at both ends, the crack which is present in the element with the lower bending rigidity has no affect on the bifurcation load at high $\mu_{a}$,

- for each of the considered systems, the magnitude of the bending rigidity asymmetry factor as a function of the crack size at which the bifurcation load is the highest can be found,

- the magnitude of $\mu_{a}$ which corresponds to the highest bifurcation load strongly depends on the Young modulus parameter $\zeta_{B}$,

- when the systems with the zero deflection angle at both ends are considered, such $\mu_{a}$ can be found at which the change of buckling shape modes can be observed; additionally, after this change, the buckling load does not depend on the crack size.

On the basis of the simulations presented in this paper the type of supports have significant influence on the sensitivity of the structure to the crack presence (taking into account the bifurcation load considered in this publication). Additionally, in the future investigations of the influence of the crack on instability of a discussed structure, different types of external load should be introduced. Structures subjected to non-conservative loads may be less vulnerable to the crack presence. A good example of such a type of load is the specific load. An introduction of the specific load modifies investigations on instability and natural vibrations (change of vibration frequencies and shape modes) regarding the classic Euler load. For the specific load (which is a 
(a)

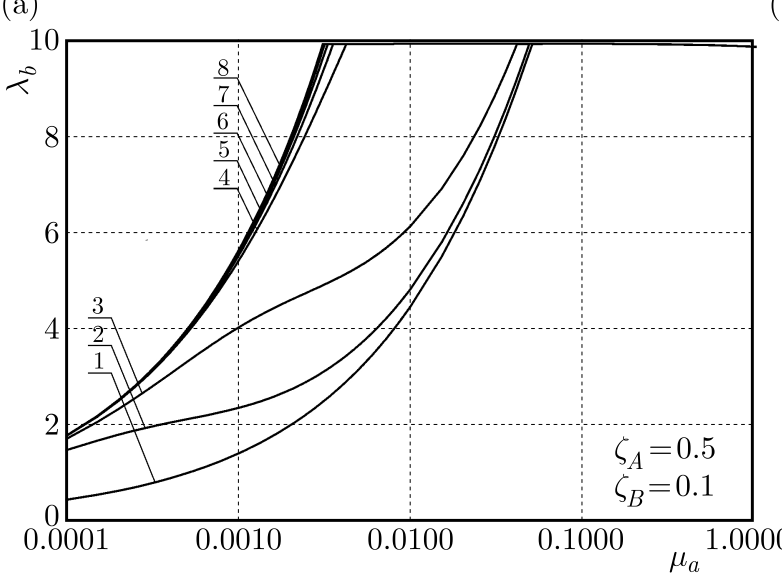

(b)

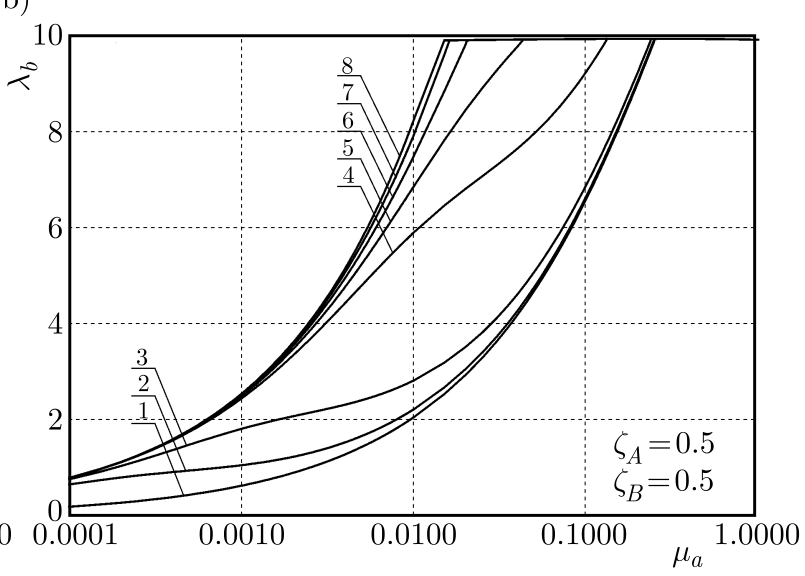

(c)

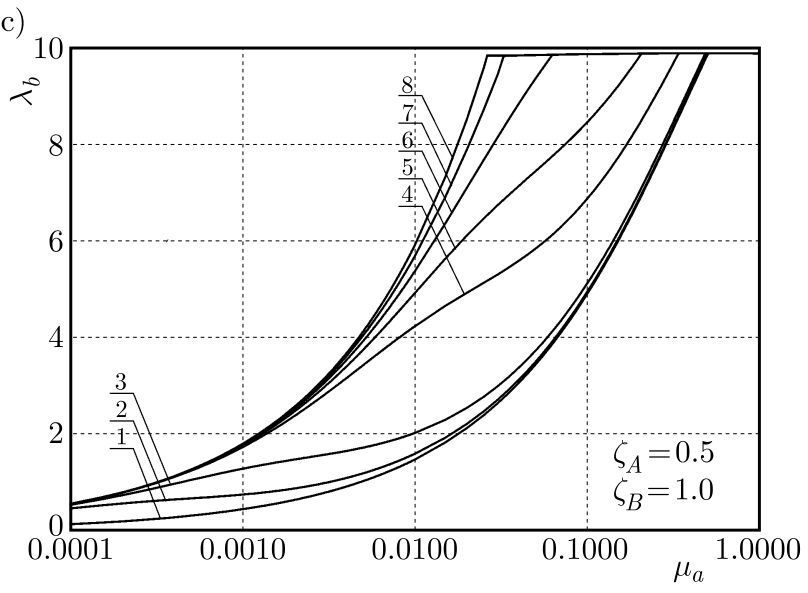

Fig. 7. A change of the bifurcation load parameter $\lambda_{b}$ of $E U 5$ column in relation to the flexural rigidity asymmetry factor $\mu_{a}$ at $\zeta_{A}=0.5$ and different crack sizes: (a) $\zeta_{B}=0.1$, (b) $\zeta_{B}=0.5$, (c) $\zeta_{B}=1$

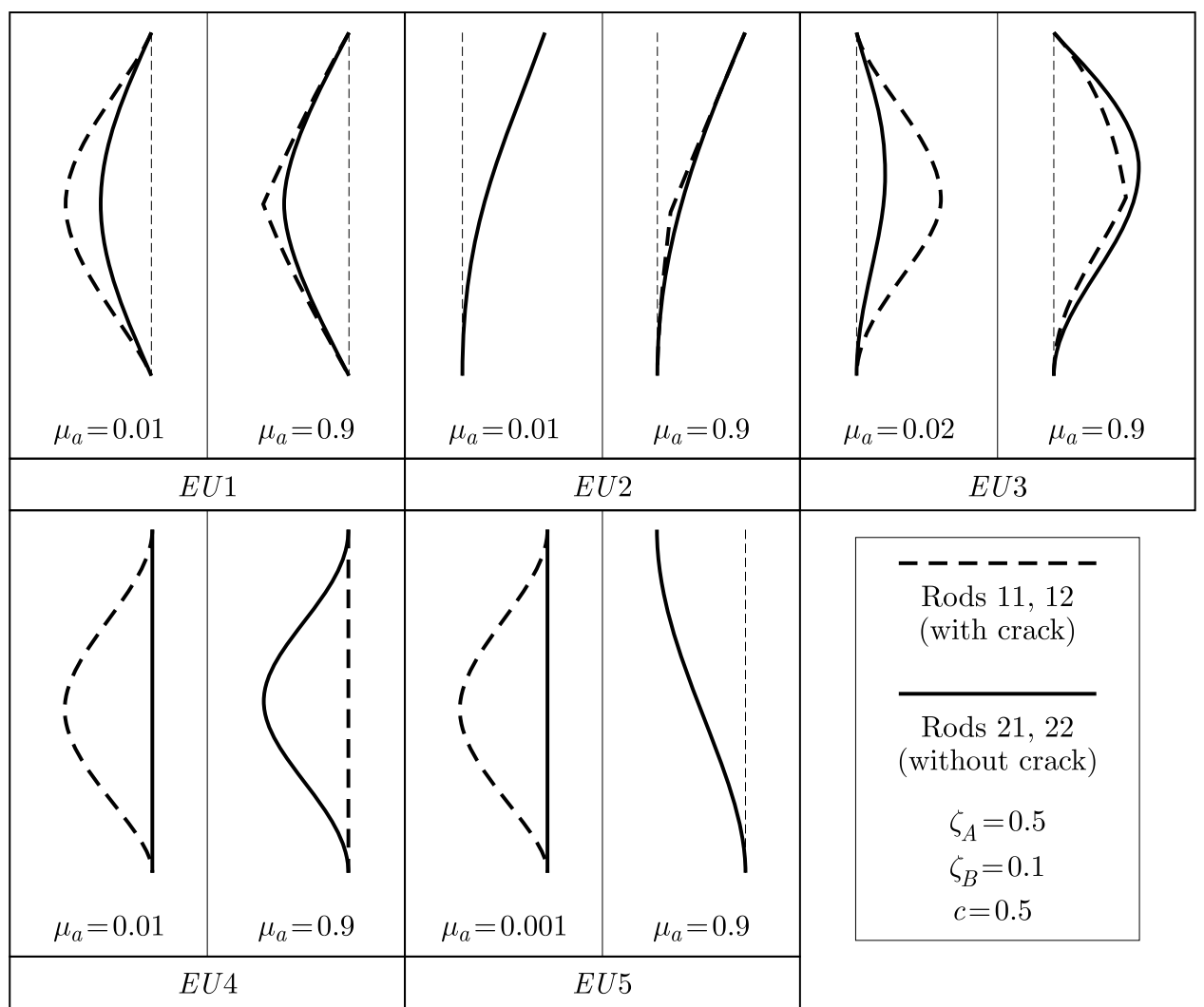

Fig. 8. Buckling mode shapes of the considered systems (EU1-EU5) for $\zeta_{B}=0.1, c=0.5$ 
practical load, see Tomski and Uzny (2008), Tomski et al. (2007, 2014)) the parameters allowing the control of the bifurcation load or the critical one as well as the natural vibration frequency can be found. It can be assumed that the parameters of the loading heads of the specific load will also affect the vulnerability of the structure to the crack presence. A continuation of this study in the mentioned way is justified and should be done in the future.

\section{Acknowledgements}

The study has been carried out within the statutory funds of the Czestochowa University of Technology (BS/PB-1-101-3020/11/P).

\section{References}

1. Anifantis N., Dimarogonas A., 1983, Stability of column with a single crack subjected to follower and vertical loads, International Journal of Solids and Strucutres, 19, 4, 281-291

2. Bochenek B., ŻycZKowski M., 2004, Analytical approach to optimization of columns for postbuckling behavior, Structural and Multidisciplinary Optimization, 28, 252-261

3. Chandros T.G., Dimarogonas A.D., Yao J., 1998, A continuous cracked beam vibration theory, Journal of Sound and Vibration, 215, 1, 17-34

4. Chati M., Rand R., Mukherjee S., 1997, Modal analysis of a cracked beam, Journal of Sound and Vibration, 207, 2, 249-270

5. Gajewski A., Życzkowski M., 1970, Optimal design of elastic columns subject to the general conservative behaviour of loading, Journal of Applied Mathematics and Mechanics, 21, 806-818

6. Ghadami A., Maghsoodi A., Miradamadi H.R., 2013, A new adaptable multiple-crack detection algorithm in beam-like structures, Archives of Mechanics, 65, 6, 469-483

7. Godley M.H.R., Chilver A.H., 1970, Elastic buckling of overbraced frame, International Journal of Mechanical Sciences, 12, 4, 238-246

8. HJelmstad K.D., Shin S., 1996, Crack identification in a cantilever beam from modal response, Journal of Sound and Vibration, 198, 5, 527-545

9. KIM K.-H., KIM J.-H., 2000, Effect of a crack on the dynamic stability of a free-free beam subjected to a follower force, Journal of Sound and Vibration, 233, 1, 119-135

10. Krawczuk M., 1992, Finite Timoshenko-type beam element with a crack, Engineering Transections, 40, 2, 229-248

11. KukLa S., 2009, Free vibrations and stability of stepped columns with cracks, Journal of Sound and Vibration, 319, 1301-1311

12. Lueschen G.G.G., Bergman L.A., Mcfarland D.M., 1996, Green's functions for uniform Timoshenko beams, Journal of Sound and Vibration, 194, 1, 93-102

13. Masoud S., Jarrah M. A., Al-MaAmory M., 1999, Effect of crack depth on the natural frequency of a prestressed fixed-fixed beam, Journal of Sound and Vibration, 214, 2, 201-212

14. NARKIS Y., 1994, Identification of crack location in vibrating simply supported beams, Journal of Sound and Vibration, 172, 4, 549-558

15. Ostachowicz W. M., Krawczuk M., 1991, Analysis of the effect of cracks on the natural frequencies of a cantilever beam, Journal of Sound and Vibration, 150, 2, 191-201

16. Rizos P.F., Aspragathos N., Dimarogonas A.D., 1990, Identification of crack location and magnitude in a cantilever beam from the vibration modes, Journal of Sound and Vibration, 138, $3,381-388$

17. Shen M.-H.H., TAYlor J.E., 1991, An identification problem for vibrating cracked beams, Journal of Sound and Vibration, 150, 3, 457-484 
18. Sokó£ K., 2014, Linear and nonlinear vibrations of a column with an internal crack, Journal of Engineering Mechanics, 140, 5

19. SokóŁ K., UZny S., 2015, Instability and vibration of multi-member columns subjected to Euler's load, Archive of Applied Mechanics, DOI 10.1007/s00419-015-1068-6

20. Tomski L., Szmidla J., Uzny S., 2007, The local and global instability and vibration of systems subjected to non-conservative loading, Thin-Walled Structures, 45, 10-11, 945-949

21. Tomski L., Szmidla J., Uzny S., 2014, Active and passive specific loads with respect to the stability and free vibrations of columns, Journal of Engineering Mechanics, 140, 1, 193-205

22. Tomski L., Uzny S., 2008, Free vibration and the stability of a geometrically non-linear column loaded by a follower force directed towards the positive pole, International Journal of Solids and Structures, 45, 1, 87-112

23. UZNY S., 2011a, Free vibrations of an elastically supported geometrically nonlinear column subjected to a generalized load with a force directed toward the positive pole, Journal of Engineering Mechanics, 137, 11, 740-748

24. UZNY S., 2011b, Local and global instability and vibrations of a slender system consisting of two coaxial elements, Thin-Walled Structures, 49, 618-626

25. Zamorska I., Cekus D., Miara M., 2015, Effect of crack parameters on free vibrations of the Berrnoulli-Euler beam, Journal of Applied Mathematics and Computational Mechanics, 14, 4, 167-174.

26. Zhang W., Wang Z., Ma H., 2009, Crack identification in stepped cantilever beam combining wavelet analysis with transform matrix, Acta Mechanica Solida Sinica, 22, 4, 360-368 